\title{
Hemangioma cavernoso intraóseo craneal
}

\author{
R. Carrasco-Moro; E. García-Navarrete; M. Navas-García; M. Adrados de Llano* y R. García de Sola
}

Servicios de Neurocirugía y de Anatomía Patológica*. Hospital Universitario La Princesa. Madrid.

\section{Resumen}

Introducción. Los hemangiomas cavernosos son tumores benignos que excepcionalmente afectan los huesos craneales. En ausencia de signos radiológicos típicos, frecuentemente son intervenidos bajo la sospecha de otro tipo de neoplasia ósea, obteniéndose el diagnóstico únicamente tras el procedimiento quirúrgico.

Caso clínico. Presentamos el caso de una paciente de 52 años con una tumoración indolora del hueso frontal derecho, de lento crecimiento y características osteolíticas desde el punto de vista neurorradiológico. Para descartar un origen metastásico, se llevó a cabo un estudio oncológico sistémico, sin hallazgo de neoplasia primaria. Finalmente, la lesión fue extirpada en bloque mediante craniectomía, seguido de craneoplastia. El diagnóstico anatomo-patológico fue hemangioma cavernoso intraóseo.

Conclusión. A pesar de su baja frecuencia, el diagnóstico de hemangioma cavernoso intraóseo debe ser considerado ante la presencia de una tumoración craneal de lento crecimiento y características osteolíticas en las pruebas de neuroimagen. El tratamiento de elección consiste en la resección completa de la lesión mediante craniectomía, incluyendo márgenes de seguridad de hueso sano.

PALABRAS CLAVE: Cavernoma. Hemangioma cavernoso. Tumor craneal.

Cavernous haemangioma of the skull

\section{Summary}

Introduction. Cavernous haemangiomas are benign tumours that rarely affect the skull. A correct suspicion diagnosis is seldom obtained when typical radiological signs are lacking. In this way a definite diagnosis is only

Recibido: 8-09-08. Aceptado: 11-11-08 obtained after a surgical procedure in most cases.

Case report. A 52-year-old female presented a painless, slow-growing tumoration in her right forehead. Skull CT showed an osteolytic lesion located within the right frontal bone. On suspicion of a metastatic origin of the lesion, a sistemic research for a primary tumour was performed without significative findings. Finally, en bloc resection of the lesion was performed followed by cranioplasty. Microscopically, the lesion proved to be a cavernous haemangioma of the frontal bone.

Conclusion. Despite their low frequency, cavernous haemangiomas must be included in the differential diagnosis of slow-growing osteolytic lesions located within the skull. The elective treatment of this tumours includes a complete resection by craniectomy, with safe bony margins.

KEY WORDS. Cavernoma. Cavernous haemangioma. Skull tumours.

Introducción

Los hemangiomas son tumores benignos que se originan en los vasos intrínsecos de los tejidos. Histológicamente, se clasifican en cavernosos y capilares, dependiendo de los vasos dominantes, aunque existen formas mixtas. Cuando afectan al tejido óseo, la columna vertebral es su localización preferente, seguida de los huesos craneales ${ }^{10}$. Los angiomas cavernosos intraóseos constituyen un diagnóstico poco frecuente, pues representan solamente un $0,7 \%$ de los tumores del hueso, y alrededor de un $10 \%$ cuando se consideran los tumores de cráneo de forma global ${ }^{6}$. A continuación, presentamos un caso de cavernoma intraóseo craneal tratado en nuestro Servicio, incluyendo una discusión del mismo a partir de una revisión de la literatura científica.

\section{Caso clínico}

Se trata de una paciente de 52 años, sin antecedentes de interés, remitida a nuestro Servicio para valoración qui- 

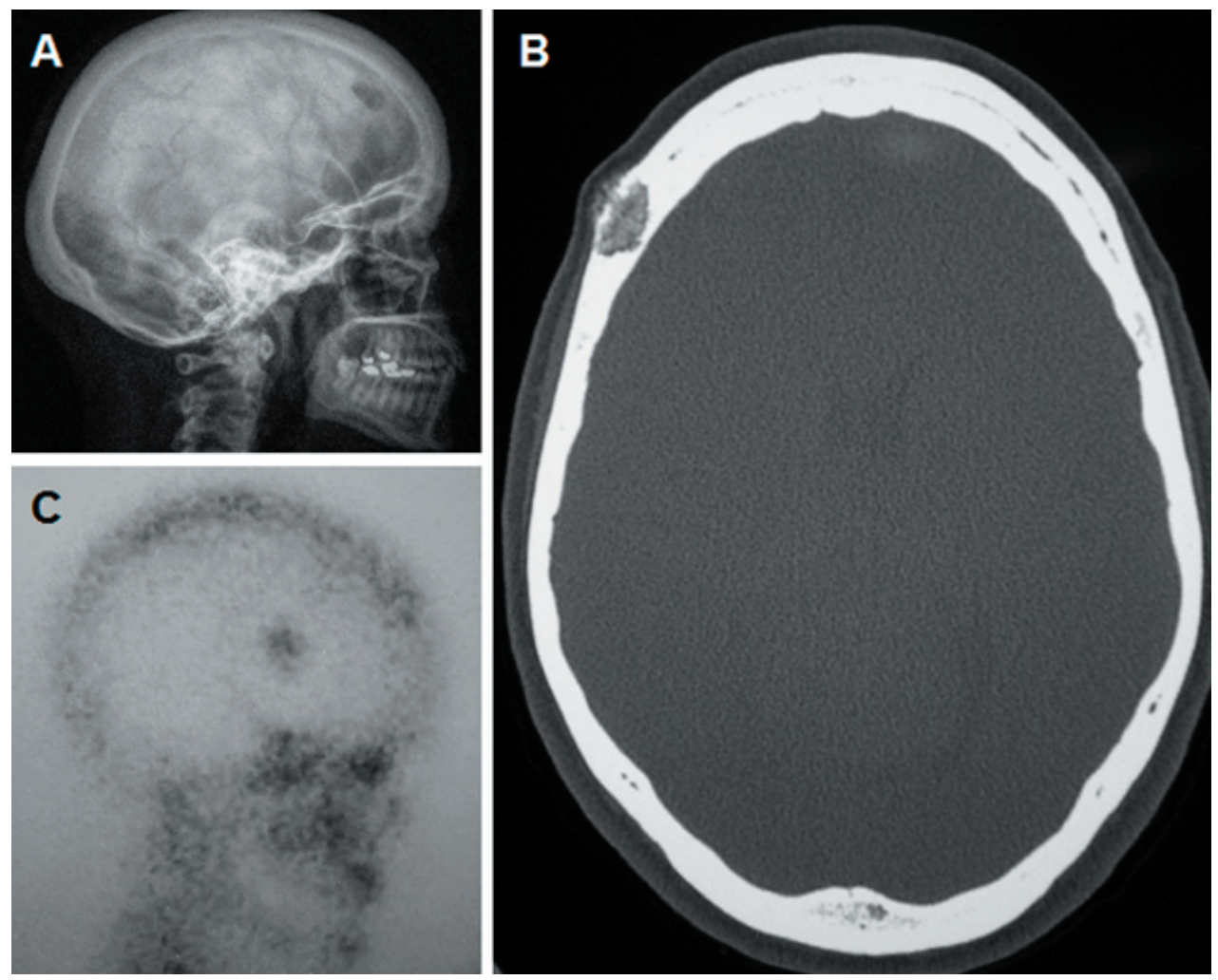

Figura 1. A) Radiografía simple de cráneo, en proyección lateral, que muestra una lesión radiolúcida, redondeada, de bordes bien definidos, localizada en el hueso frontal; B) En la TAC con ventana ósea, la lesión presenta áreas de densidad heterogénea en su interior. Invade el díploe y erosiona las tablas interna y externa del hueso frontal derecho, expandiendo ésta última; C) Gammagrafía con HDP-Tc99M, evidenciando un depósito patológico que se corresponde topográficamente con la lesión descrita.

rúrgica. Presentaba, desde hacía cuatro años, una pequeña tumoración en la porción derecha de la frente, que había aumentando lentamente de tamaño. Durante la exploración se palpaba una masa dura, indolora y no movilizable, bajo una piel de aspecto normal.

La radiografía simple (figura 1A) y la TAC (figura 1B) de cráneo mostraron una lesión intraósea frontal derecha de características osteolíticas. El diagnóstico diferencial radiológico incluyó metástasis, mieloma y hemangioma. Los estudios sistémicos de rastreo tumoral (hemograma, frotis hematológico, marcadores tumorales, proteinograma y TAC cervico-toraco-abdominal) fueron negativos. Se realizó una gammagrafía ósea con HDP-Tc99M, demostrándose un depósito redondeado en el área de la lesión (figura 1C). La punción-aspiración percutánea con aguja fina de la tumoración resultó no concluyente para el diagnóstico, al obtenerse únicamente fragmentos hemáticos.

Finalmente, se decidió intervenir quirúrgicamente a la paciente en base a la progresión clínica de la lesión, con sus implicaciones estéticas, así como para obtener un diagnóstico histológico definitivo. Durante el acto quirúrgico, se identificó una tumoración dependiente del hueso, con múlti- ples canales vasculares dilatados en su seno, que expandía la tabla externa (figuras 2A y 2B). Para evitar la manipulación de la lesión, se decidió incluirla en una pieza de craniectomía con un margen circunferencial de $1 \mathrm{~cm}$. de hueso aparentemente sano. El defecto óseo resultante fue reconstruido mediante una plastia craneal de metilmetacrilato, que se fijó al hueso circundante con miniplacas de Titanio. El periodo postoperatorio transcurrió sin incidencias. El diagnóstico anatomopatológico definitivo fue de hemangioma cavernoso intraóseo (figura 2C).

\section{Discusión}

\section{Características clínicas}

El primer caso de hemangioma cavernoso craneal fue descrito por Toynbee en $1845^{17}$. Desde entonces, la mayoría de las publicaciones en la literatura científica se han presentado en formato de caso clínico único, exceptuando dos revisiones de amplia casuística que constituyen las principales referencias sobre esta entidad ${ }^{1,6}$.

Los cavernomas craneales pueden aparecer a cualquier 

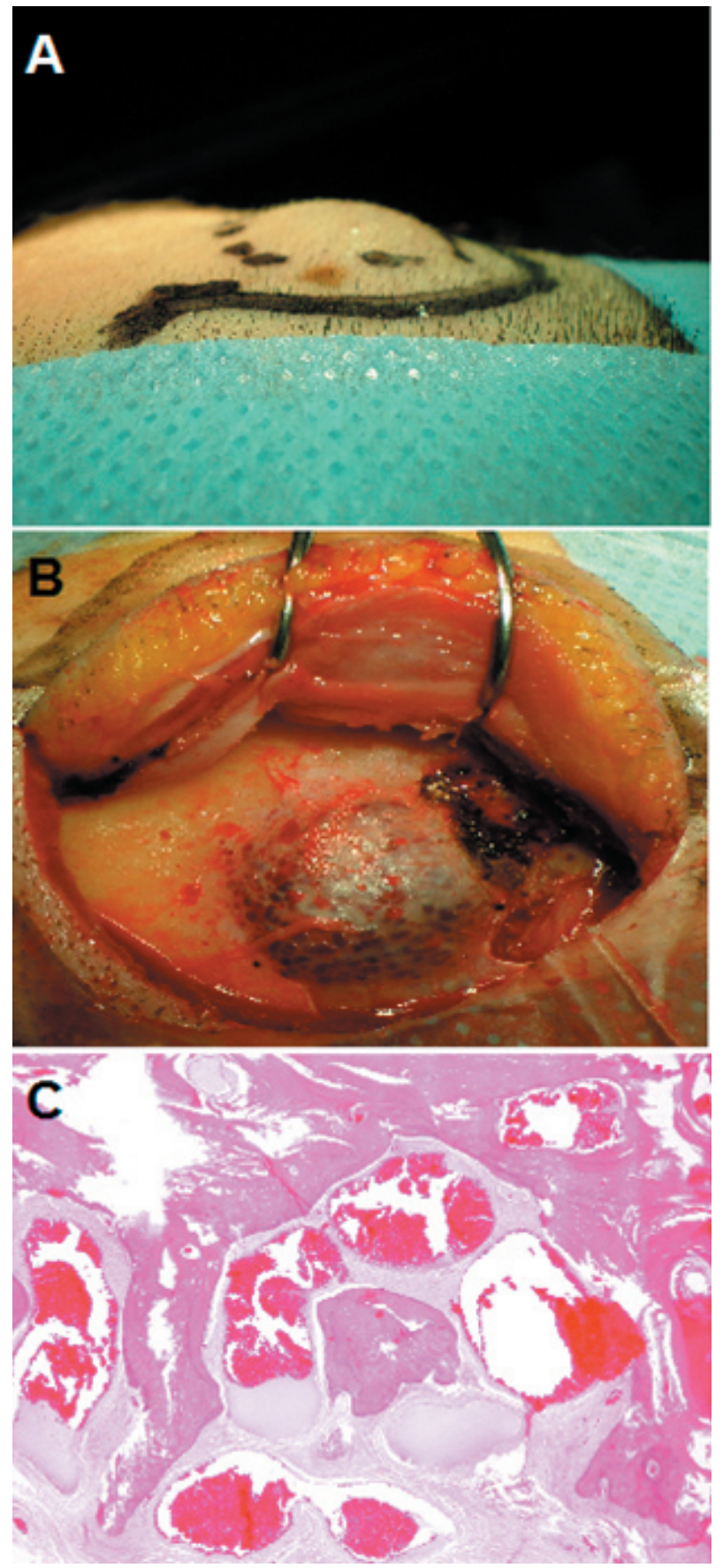

Figura 2. A) Tumoración frontal derecha de $2 \mathrm{~cm}$. de diámetro: diseño de la incisión quirúrgica; B) Bajo una piel y un periostio intactos, se identifica una lesión expansiva dependiente del hueso, con espacios vasculares en su seno; C) Desde el punto de vista microscópico, la lesión se compone de estructuras vasculares dilatadas de paredes finas, localizadas dentro del espacio medular entre trabéculas óseas persistentes. Estas estructuras están tapizadas por células endoteliales aplanadas dispuestas en una capa única. (H-E, x40).
2009 20: 559-562

edad, pero afectan sobre todo a mujeres con edades comprendidas entre la segunda y cuarta décadas ${ }^{1,6}$. Habitualmente son lesiones únicas, aunque se han descrito casos de cavernomas craneales múltiples ${ }^{10}$. La calota es la región más frecuentemente afectada, fundamentalmente a nivel del hueso frontal, seguido del temporal y parietal ${ }^{6}$. Los hemangiomas cavernosos del cráneo suelen presentar un tamaño en el momento del diagnóstico que oscila entre los 15 y $25 \mathrm{~mm}$., aunque se han descrito lesiones de hasta $8 \mathrm{~cm}$. de diámetro ${ }^{2,7}$.

Los hemangiomas cavernosos pueden aparecer prácticamente en cualquier localización anatómica craneal, propiedad que va a condicionar la aparición de una amplia variedad de manifestaciones clínicas. En el caso de los cavernomas de la calota, la presentación típica viene dada por la presencia de una masa dura, indolora, que aumenta lentamente de tamaño bajo una piel suparayacente intacta ${ }^{10}$. El patrón de crecimiento de estos tumores suele ser exofítico, aunque se han descrito casos con extensión intracraneal, pudiendo asociar un sangrado epidural o subdural ${ }^{5,7}$. Otras presentaciones menos frecuentes incluyen exoftalmos y/o diplopia en el caso de aquellos hemangiomas cavernosos de localización orbitaria, así como afectación de pares craneales cuando los cavernomas implican los huesos de la base del cráneo ${ }^{4}$.

\section{Diagnóstico}

La TAC craneal con ventana ósea constituye la modalidad diagnóstica de elección, ya que supera en sensibilidad a la radiografía simple y permite definir el hueso de forma superior a la RM. La imagen característica consiste en una lesión lítica, oval o redondeada, bien delimitada, con trabéculas que irradian desde un centro común en su interior en los cortes tangenciales, dando en ocasiones una apariencia de panalización en los cortes axiales ${ }^{11,12,16}$. Frecuentemente invade y expande la tabla externa, respetando el periostio. Habitualmente no se identifican signos de hiperostosis reactiva en sus márgenes ${ }^{6}$. En la RM, la lesión suele ser de intensidad mixta, predominantemente isointensa o hiperintensa en secuencia T1, hiperintensa en $\mathrm{T} 2$, y con captación de contraste tras la administración de Gadolinio $^{6,12,16}$.

El diagnóstico diferencial incluye otras lesiones expansivas óseas de lento crecimiento, como el osteoma, quiste óseo aneurismático, tumor de células gigantes, displasia fibrosa, sarcoma, meningioma, metástasis, enfermedad de Paget, quiste dermoide y mieloma múltiple. Habitualmente el diagnóstico definitivo suele obtenerse únicamente tras una intervención quirúrgica, que constituye al mismo tiempo una maniobra diagnóstica y terapéutica $^{6,10}$. La realización de una punción-aspiración con aguja fina raramente está indicada durante el manejo diagnóstico 
de las neoplasias de la bóveda craneal, ya que sus características morfológicas y topográficas las hacen habitualmente susceptibles de un tratamiento quirúrgico definitivo, independientemente de sus sustrato patológico ${ }^{6}$.

\section{Tratamiento y pronóstico}

La historia natural de estas lesiones no ha sido todavía descrita. Considerando que los hemangiomas cavernosos óseos crecen de forma progresiva, habitualmente se recomienda su tratamiento quirúrgico, ya que aquel fenómeno implica un empeoramiento del cuadro clínico, incluyendo desde formas banales con implicaciones puramente cosméticas hasta sucesos relevantes como hemorragias o lesión de nervios craneales, dependiendo de la localización de la lesión 6 .

La primera resección con éxito de un angioma craneal fue llevada a cabo por Pilcher en 1894. Previamente, en 1845, Ehrmann ya lo había intentado, pero el paciente falleció de meningitis postquirúrgica ${ }^{14,15}$. En 1923, Cushing diseñó el que representa el tratamiento de elección de los hemangiomas cavernosos craneales hasta nuestros días: la resección en bloque de la lesión, incluyendo un margen circunferencial de hueso sano ${ }^{19}$. Esta técnica evita una manipulación intratumoral innecesaria, que conlleva un riesgo de sangrado abundante en el caso de lesiones de gran tamaño. Por otra parte, se elude la posibilidad de recidiva al incluir un margen de seguridad ${ }^{13,18}$.

La radioterapia se debe reservar para aquellas lesiones que se consideran irresecables o en el caso de tumores recidivantes. Esta modalidad terapéutica detiene el crecimiento tumoral y disminuye su vascularización, pero no modifica el tamaño de la lesión y acarrea el riesgo de malignización o aparición de malignancias de novo ${ }^{3,8,9}$.

\section{Conclusión}

Los hemangiomas cavernosos craneales son tumoraciones benignas que, en ausencia de características radiológicas típicas, habitualmente son intervenidas quirúrgicamente bajo la sospecha de otro tipo de neoplasia ósea. Su tratamiento de elección es una resección completa mediante craniectomía, incluyendo márgenes óseos sanos de seguridad.

\section{Bibliografía}

1. Barnes, L.: Solitary hemangioma of bone. En Barnes L (ed). Surgical pathology of the head and neck, vol 1. New York; Dekker, 1985; pp. 932-936.

2. Dogan, S., Kocaeli, H., Sahin, S., Korfali, E., Saraydaroglu, O.: Large cavernous hemangioma of the frontal bone. Neurol Med Chir (Tokyo) 2005; 45: 264-267.

3. Fredrickson, J.M., Haight, J.S.H., Noyek, A.M.: Radia- tion induced carcinoma in a hemangioma. Otolaryngol Head Neck Surg 1979; 87: 584-586.

4. González-Darder, J.M., Pesudo-Martínez, J.V.: Parálisis facial por angioma cavernoso del peñasco. Caso clínico. Neurocirugía 2007; 18: 44-46.

5. Gottfried, O.N., Gluf, W.M., Schimdt, M.H.: Cavernous hemangioma of the skull presenting with subdural hematoma. Neurosurg Focus 2004; 17: 1-4.

6. Heckl, S., Aschoff, A., Kunze, S.: Cavernomas of the skull: review of the literature 1975-2000. Neurosurg Rev 2002; 25: 56-62.

7. Hernández-Borroto, C.E., Amado-Donestévez, A., Vaquer-Fernández, J. E., Medrano-Plana, Y., Ruíz-Martín, M.: Hemangioma cavernoso gigante de la bóveda craneal. Rev Neurol 2004; 38: 799-800.

8. Jaffe, H.L.: Tumors and tumorous conditions of the bones and joints. Philadelphia; Lea and Febiger, 1958; pp. 224-239.

9. Mangham, C.A., Carberry, J.N., Brackmann, D.E.: Management of intratemporal vascular tumors. Laryngoscope 1981; 91: 867-876.

10. Naama, O., Gazzaz, M., Akhaddar, A., et al.: Cavernous hemangioma of the skull: 3 case reports. Surg Neurol 2008 (in press).

11. Nasser, K., Nakamasa, H., Kurosaki, K., et al.: Intraosseous cavernous hemangioma of the frontal bone. Neurol Med Chir (Tokyo) 2007; 47: 506-508.

12. Nguyen, B.D., McNaughton, D.: Nuclear imaging of a tender skull mass. AJN 2007; 189: S61- S63.

13. Peterson, D.L. Murk, S.W., Story, J.L.: Multiple cavernous hemangiomas fo the skull. Report of a case and review of the literature. Neurosurgery 1992; 30: 778-782.

14. Pilcher, L.S.: Venous tumour of the diploe. Trans Am Surg Assoc 1894; 2: 283-285.

15. Schöne, G.: Über einen Fall von myelogenem Hemangiom des Os occipitale. Beiträge zur pathologischen Anatomie und zur allgemeinen. Pathologie 1905; (Suppl) 7: 685-701.

16. Suzuki, Y., Ikeda, H., Mutsamoto, K.: Neuroradiological features of intraosseous cavernous hemangioma. Neurol Med Chir (Tokyo) 2001; 41: 279-282.

17. Toynbee, J.: An account of two vascular tumors developed in the substance of bone. Lancet 1845; 2: 676.

18. Wold, L.E., Swee, R.G., Sim, F.H.: Vascular lesions of bone. Pathol Annu 1985; 2: 101-109.

19. Zucker, J.J., Levine, M.R., Chu, A.: Primary intraosseous hemangioma of the orbit: report of a case and review of the literature. Ophtalmic Plast Reconstr Surg 1989; 5: 247-255.

Carrasco-Moro, R.; García-Navarrete, E.; Navas-García, M.; Adrados de Llano, M.; García de Sola, R.: Hemangioma cavernoso intraóseo craneal. Neurocirugía 2009; 20: 559-562.

Correspondencia postal: Rodrigo Carrasco Moro. H. U. La Princesa. Servicio de Neurocirugía. C/ Diego de León 62. 28006. Madrid. 